\title{
GENERATION AND CHARACTERIZATION OF CELL LINES STABLY EXPRESSING DIFFERENT ISOFORMS OF KCNQ POTASSIUM CHANNELS SUITABLE FOR DRUG SCREENING
}

I. Coletta, B. Garofalo, R. Ombrato, C. Milanese, F.P. Di Giorgio, S.Tongiani

Angelini RR\&D (Regulatory, Research \& Development) - Angelini S.p.A. P.le della Stazione snc, $00071 \mathrm{~S}$ Palomba-Pomezia (Rome), Italy

www.angelinipharma.com - isabella.coletta@angelinipharma.com

\section{INTRODUCTION}

Voltage-gated Kv7.2 and Kv7.3 channels, belonging to the Kv7 or KCNQ potassium channel family, are responsible for regulation of $\mathrm{M}$-currents, ${ }^{1}$ a low-threshold, depolarization-activated $\mathrm{K}+$ current, uniquely suited to suppress bursting and epileptiform activity, while permitting maintenance of responses to ordinary excitatory inputs. ${ }^{2}$ Pharmacological activation of such channels may be a strategy to treat hyperexcitability conditions such as epilepsy and neuropathic pain. After discontinuation of the anticonvulsant retigabine, ${ }^{3}$ the identification of novel $\mathrm{Kv7.2} / \mathrm{Kv7.3}$ channel activators is an unmet medical need with promising therapeutic efficacy. In the present work, we show the generation of cell lines stably expressing different isoforms of KCNQ potassium channels and their characterization in functional assays to allow generation of High-Throughput Screening (HTS) data for the identification of novel Kv7.2/Kv7.3 (KCNQ 2/3) openers.

The human genes for Kv7.2 and Kv7.3 channel subunits were synthetized and cloned in suitable mammalian expression vectors. Kv7.2 and Kv7.3 constructs were stably transfected into CHO K1 cell line to generate 3 cell lines expressing the different Kv7.2/Kv7.3 hetero-tetramers, Kv7.2 and Kv7.3 homo-tetramers.

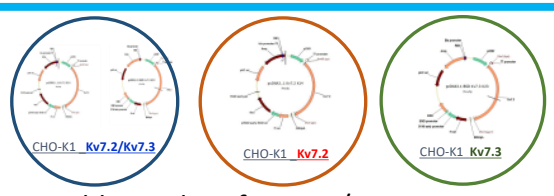

The transfected cell lines were used to develop 2 different assays suitable to identify Kv7.2/Kv7.3 openers: A) Fluorescence-based FLIPR-TETRA assay using a Thallium sensitive dye; B) Q-Patch voltage clamp assay. Retigabine and ICA-069673 as reference activators and TEA-Cl as reference inhibitor were used.

\section{FLUORESCENCE-BASED SCREENING ASSAY TO DISCOVER NOVEL KCNQ 2/3 CHANNEL OPENERS}
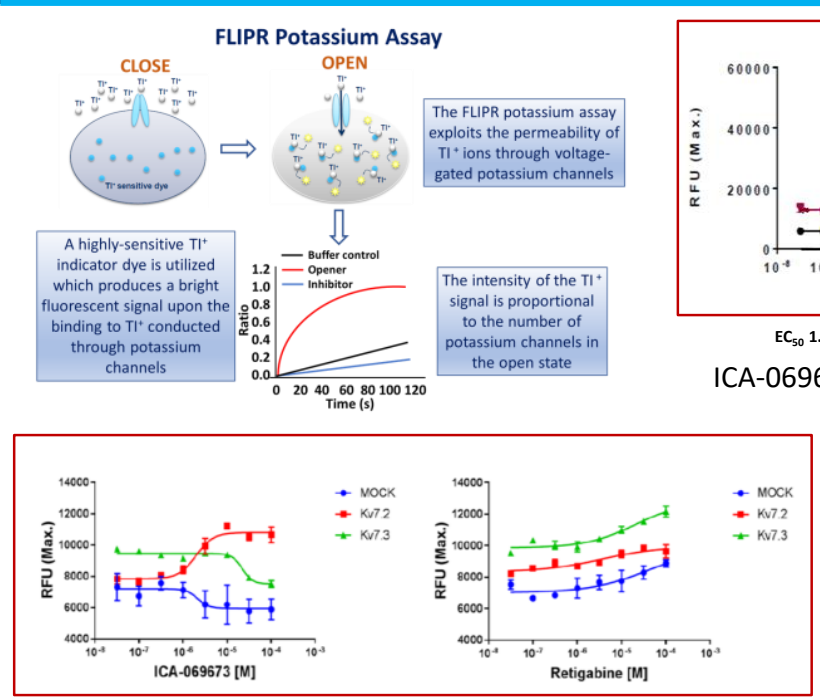

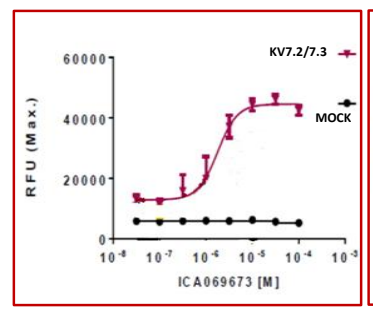

$\mathrm{EC}_{50}$ 1.78E-06

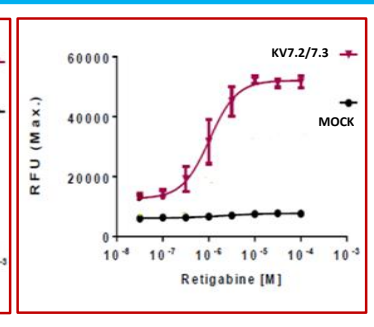

$\mathrm{EC}_{50} 1.04 \mathrm{E}-06$

ICA-069673 and retigabine dose-response curves in Kv7.2/Kv7.3 and MOCK cells.

ICA-069673 was chosen as reference opener for Kv7.2.

Retigabine was selected as reference opener for Kv7.3.

A

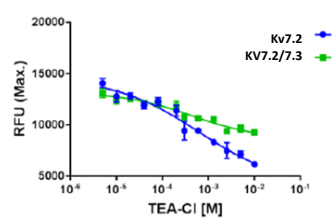

B

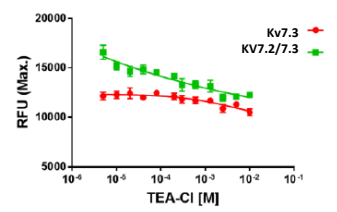

A. A reduction of ICA-069673-induced signal by TEA-Cl treatment can be appreciated in both Kv7.2 and Kv7.2/Kv7.3.

B. A reduction of retigabine-induced signal by TEA-Cl treatment can be appreciated only in Kv7.2/Kv7.3 but not in the homomeric Kv7.3.

The functional discrimination between isoforms at FLIPR-TETRA indicates that the two subunits coexist in the heteromeric clone. 


\section{PATCH-CLAMP BASED SCREENING ASSAY TO DISCOVER NOVEL KCNQ 2/3 CHANNEL OPENERS}

Q-Patch_Voltage clamp protocol:

cells were held at $-80 \mathrm{mV}$ and the Kv current was evoked by 1-1.5 sec-long depolarization steps $(+10 \mathrm{mV}$ ) ranging from $-100 \mathrm{mV}$ to $+20 \mathrm{mV}$, followed by a depolarization step at $0 \mathrm{mV}(200 \mathrm{msec})$ and back to $-80 \mathrm{mV}(1 \mathrm{~min})$. At $0 \mathrm{mV}$ the current was measured as the state of the channel exactly before the depolarization at each $\mathrm{V}$ imposed in the conditioning phase.

1. Current densities $(\mathrm{pA} / \mathrm{pF})$ at the end of each depolarization were measured. This value is then plotted against its own voltage step to obtain the $\mathrm{I} / \mathrm{V}$ relationship.

2. Instantaneous current at $0 \mathrm{mV}$ is plotted against the preconditioning voltage step value then fitted with a Boltzmann equation and normalized to the maximal (extrapolated) value to obtain the activation curve. The activation curve represents the probability of channel opening from 0 to 1 on $Y$ scale.

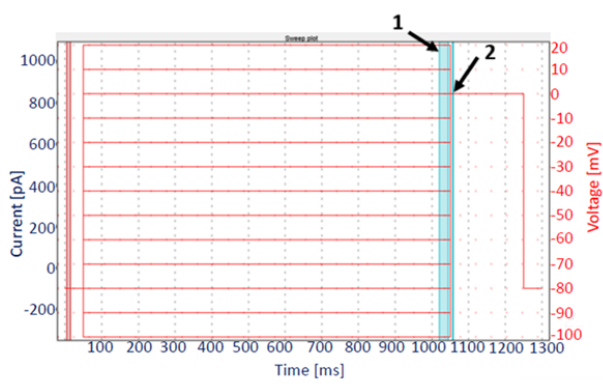

Current traces were analyzed in two areas, as pointed by the numbers 1 (late current) and 2 (tail current)
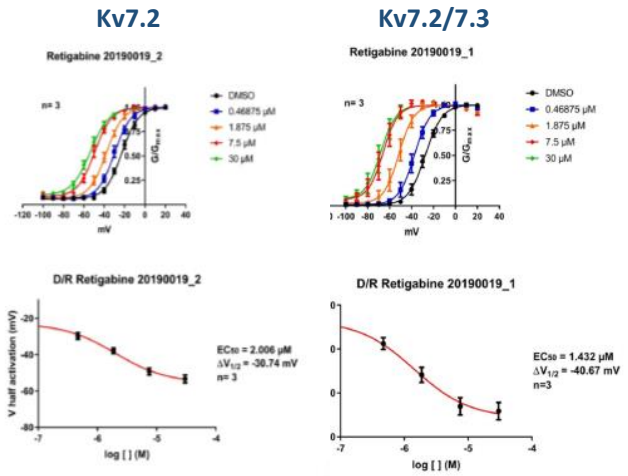

The $\underline{\Delta V_{1 / 2}}$ value is a measure of the drug efficacy
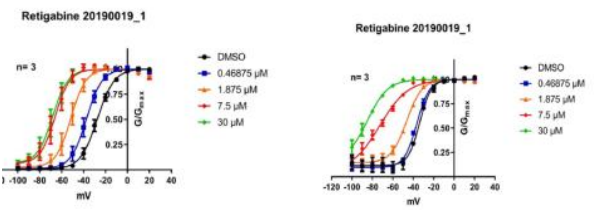

The $V_{1 / 2}(m V)$ value representing the voltage at which the channels are activated at $50 \%$.

$\Delta \mathrm{V}_{1 / 2}=\mathrm{V}_{1 / 2}(@ 30 \mu \mathrm{M})-\mathrm{V}_{1 / 2}$ (DMSO)

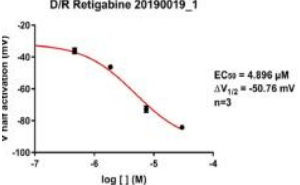

The value of $\mathrm{EC}_{50}$ is extrapolated from the saturation curve.

The $\underline{E C}_{50}$ value is a measure of the drug potency

Functional discrimination between isoforms at Q-Patch16 $\mathrm{X}$ with TEA-Cl

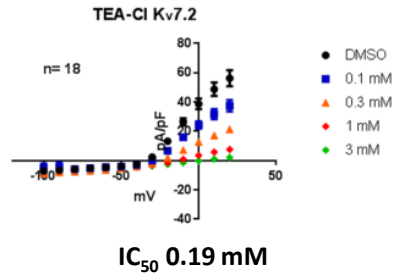

TEA-Cl causes a complete inhibition of the $\mathrm{K}+$ current in $\mathrm{Kv} 7.2$ at $3 \mathrm{mM}$

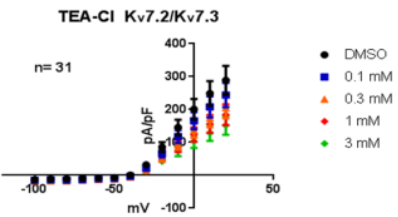

$\mathrm{IC}_{50} 1.5 \mathrm{mM}$

in Kv7.2/Kv7.3 an inhibition of $~ 50 \%$, confirming the presence of the heteromeric isoform

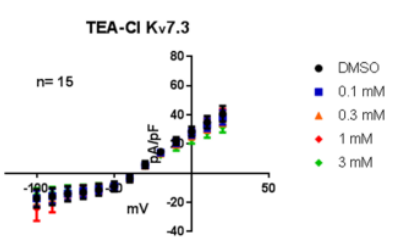

$\mathrm{IC}_{50} \mathrm{nc}$

almost no effect of TEA-Cl is observed in the isoform Kv7.3

A proprietary library of 35000 compounds was tested against CHO-K1 Kv7.2/Kv7.3 cells in the FLIPR based HTS. 30 hits identified, with micromolar activity, were characterized in dose response curves in electrophysiology in an automated Q-patch $16 \mathrm{X}$ system.

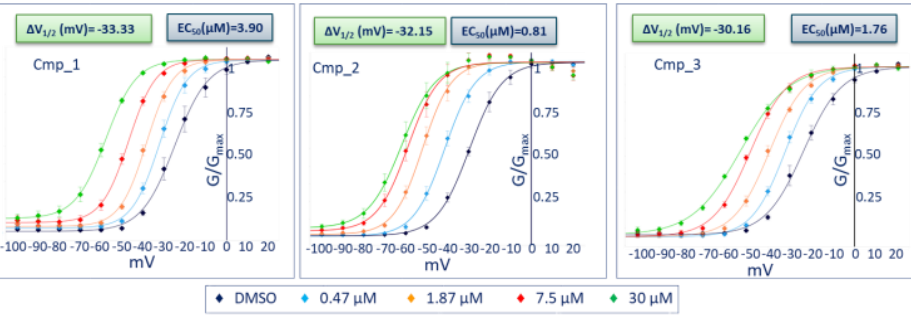

Activation curves for three representative compounds

\section{CONCLUSIONS}

Angelini has combined a fluorescent and an electrophysiological method to generate high quality data to develop a HTS campaign in order to identify novel Kv7.2/Kv7.3 openers as potential therapeutics for neurological disorders characterized by high excitability.

References: 1) Soldovieri M.V.; et al.(2011). Physiology (Bethesda), 26 (5), 365-76. 2) Rogawski M. A. et al. (2008). Curr Neurol Neurosci Rep., 8(4): 345-352. 3) M. J. Gunthorpe et al. (2012). Epilepsia, 53(3):412-424.

\section{Acknowledgments}

The authors would like to thank the Cell Biology Unit, the Hit Tailoring Solutions Unit and the Electrophysiology Unit of AXXAM S.p.A. for suggestions and experimental contribution. 